\title{
Modificação do Nódulo Sinusal Via Cateter por Energia de Radiofrequiência em Paciente com Taquicardia Sinusal Inapropriada. Avaliação dos Resultados Imediatos e Tardios
}

\author{
Fernando E. S. Cruz Fo, Ivan G. Maia, Marcio L. A. Fagundes, Silvia Boghossian, José Carlos Ribeiro \\ Rio de Janeiro, RJ
}

São apresentados os resultados imediatos e tardios, observados com a modificação do nódulo sinusal via cateter, usando-se energia de radiofreqüência, em portadora de taquicardia sinusal inapropriada. $O$ procedimento normalizou os níveis de freqüência cardíaca nas $24 \mathrm{~h}$ seguintes e após seis meses, mostrando-se, portanto, efetivo e seguro. Apesar da redução da freqüência cardíaca a níveis normais, houve manutenção dos sintomas apresentados pela paciente, sugerindo que não são decorridos, exclusivamente, do processo taquicárdico.

\section{Sinus Node Modification by Catheter Using Radiofrequency Current in a Patient with Inappropriate Sinus Tachycardia. Evaluation of Early and Late Results}

This article reports the early and late results of a patient in whom radiofrequency current was used to ablate an incessant inappropriate sinus tachycardia. During successful radiofrequency application there was a sudden change of rate and subsequent emergence of a stable rhythm with the same sinus node $P$ wave characteristics. During follow-up, normal cardiac cycles were still present after six months of the procedure, although the patient still complained of palpitations, suggesting no correlation with the heart rate.
A taquicardia sinusal inapropriada é uma arritmia rara, que ocorre em indivíduos, aparentemente, normais, caracterizada por um ritmo taquicárdico crônico ( $>3$ meses de duração), freqüência $>100$ bpm durante vigília, resposta adrenérgica exacerbada e aspecto eletrocardiográfico da onda $\mathrm{P}$, sugestivo de sua origem sinusal ${ }^{1}$. A condição leva, freqüentemente, a sintomas tipo palpitações e cansaço $^{2}$, sendo de difícil controle clínico, mostrando geralmente resposta inadequada ao uso de drogas antiarrítmicas, que reduzem a freqüência sinusal. Ignora-se o seu mecanismo de produção, podendo estar relacionado a um aumento do automatismo sinusal ou o de alguma estrutura próxima ao mesmo.

Tem sido proposta para controle da freqüência cardíaca anormal de pacientes com taquicardia sinusal inapropriada, a modificação do nódulo sinusal, através da aplicação sobre essa estrutura via cateter de energia do tipo radiofreqüência ${ }^{3,4}$. Embora os resultados sejam ainda inici-

Hospital Pró-Cardíaco / Pró-Ritmo / Ritmolab - Rio de Janeiro

Correspondência: Fernando E. S. Cruz F ${ }^{o}$ - Hospital Pró-Cardíaco - Rua Dona Mariana, 219 - 22280-020 - Rio de Janeiro, RJ

Recebido para publicação em 3/10/97

Aceito em 7/1/98 ais, parecem promissores, pelo menos no que diz respeito ao controle a curto prazo do ritmo taquicárdico.

Apresentamos os resultados imediatos e tardios observados com a modificação do nódulo sinusal via cateter, aplicando-se energia de radiofreqüência, em caso de taquicardia sinusal inapropriada, tendo sido os mesmos monitorizados por gravações de Holter com determinação dos ciclos cardíacos.

\section{Relato do Caso}

Mulher branca, de 33 anos, com manifestações crônicas de dor precordial não relacionadas aos esforços, palpitações e cansaço; classe funcional I (NYHA). No exame clínico, pressão arterial (PA) de 120/80 $\mathrm{mmHg}$, pulso radial regular, taquicárdico, com características normais. Ritmo cardíaco em dois tempos, bulhas inalteradas e ausência de sopros. Pulsos periféricos presentes e de características normais. Função tireoidiana e glicemia normais. Eletrocardiograma mostrando um ritmo de origem sinusal com ondas P com duração de 0,08 s, frequiência média de $110 \mathrm{bpm}$ e condução atrioventricular 1:1. Complexos QRS estreitos, com orientação espacial normal no plano frontal. Ondas T com alterações inespecíficas. Gravação de Holter-24h confirmou 
o ritmo sinusal durante todo o período dos registros com ausência de arritmias ativas ectópicas. Frequiência cardíaca média durante vigília de $111 \mathrm{bpm}$ e durante o sono de $91 \mathrm{bpm}$. Ecocardiograma mostrando estruturas e função ventricular normais. A paciente fazia uso inicial de propranolol com dose máxima de $120 \mathrm{mg}$ oral/dia, sem controle do ritmo anormal e dos sintomas, obtendo-se a mesma resposta com o uso de verapamil (até $240 \mathrm{mg}$ oral/dia). Com o diagnóstico de taquicardia sinusal inapropriada estabelecido e frente aos sintomas relatados, foi proposto tratamento invasivo, procedendo-se a modificação do nódulo sinusal via cateter, com o uso de energia tipo radiofreqüência.

Por técnica de cateterismo direito, foram introduzidos por via femoral, um cateter tripolar posicionado ao nível da válvula tricúspide e o cateter de ablação, posicionado em átrio direito alto. Durante estimulação atrial contínua ou varredura da diástole por extra-estímulos, havia captura do ritmo e reciclagem momentânea com parada da estimulação artificial. Após um máximo de dois ciclos, observou-se o retorno aos níveis de frequiência pré-estimulação. O tempo de recuperação e de condução sino-atrial foram normais. No mapeamento atrial alto detectou-se uma região com precocidade de $28 \mathrm{~ms}$ em relação a onda $\mathrm{P}$, localizada próximo à junção do átrio direito e veia cava superior, em região da crista terminalis. No local foram liberados um total de 225 watts de energia com um tempo total de aplicação de $4,5 \mathrm{~min}$. $\mathrm{Na} 7^{\mathrm{a}}$ aplicação houve redução súbita da freqüência da taquicardia com uma nítida modificação na seqüência de ativação atrial (fig. 1 e 2). Foram liberadas mais duas sessões de energia como reforço.

Gravações de Holter foram obtidas nas $24 \mathrm{~h}$ que precederam (pré) e sucederam (pós) o procedimento, bem como após aos seis meses. Em todos os registros foram computados os valores médios dos ciclos cardíacos das $24 \mathrm{~h}$ bem como os correspondentes à vigília (10-18h) e sono (24-8h). Esses resultados foram comparados sob o ponto de vista estatístico aplicando-se um teste de variância com valor de $\mathrm{p}<0,05$ como significativo. Considerou-se a arritmia como controlada frente a uma resposta de freqüência com ciclos médios >600ms nas $24 \mathrm{~h},>550 \mathrm{~ms}$ na vigília e >650ms durante o sono, imediatamente e seis meses após o procedimento.

No pré-procedimento, o ciclo médio das $24 \mathrm{~h}$ encontrava-se em 520,9 $989,1 \mathrm{~ms}$ com variação máxima e mínima de 725,0 e 416,0ms, respectivamente; durante vigília de $449,7 \pm 22,6 \mathrm{~ms}$ e sono de $612,1 \pm 81,7 \mathrm{~ms}$. Imediatamente após a modificação o ciclo médio das $24 \mathrm{~h}$ passou para $733,4 \pm$ 67,3ms com variação máxima e mínima de 869,0 e 583,0ms, respectivamente; durante vigília passou para 723,2 $\pm 49,7 \mathrm{~ms}$

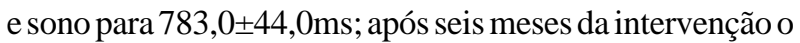

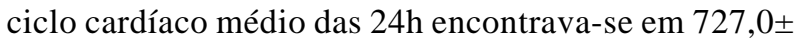
128,3ms com variação máxima e mínima de 915,0 e 504,0ms, respectivamente. Durante vigília e sono em 599,4ะ98,0ms e

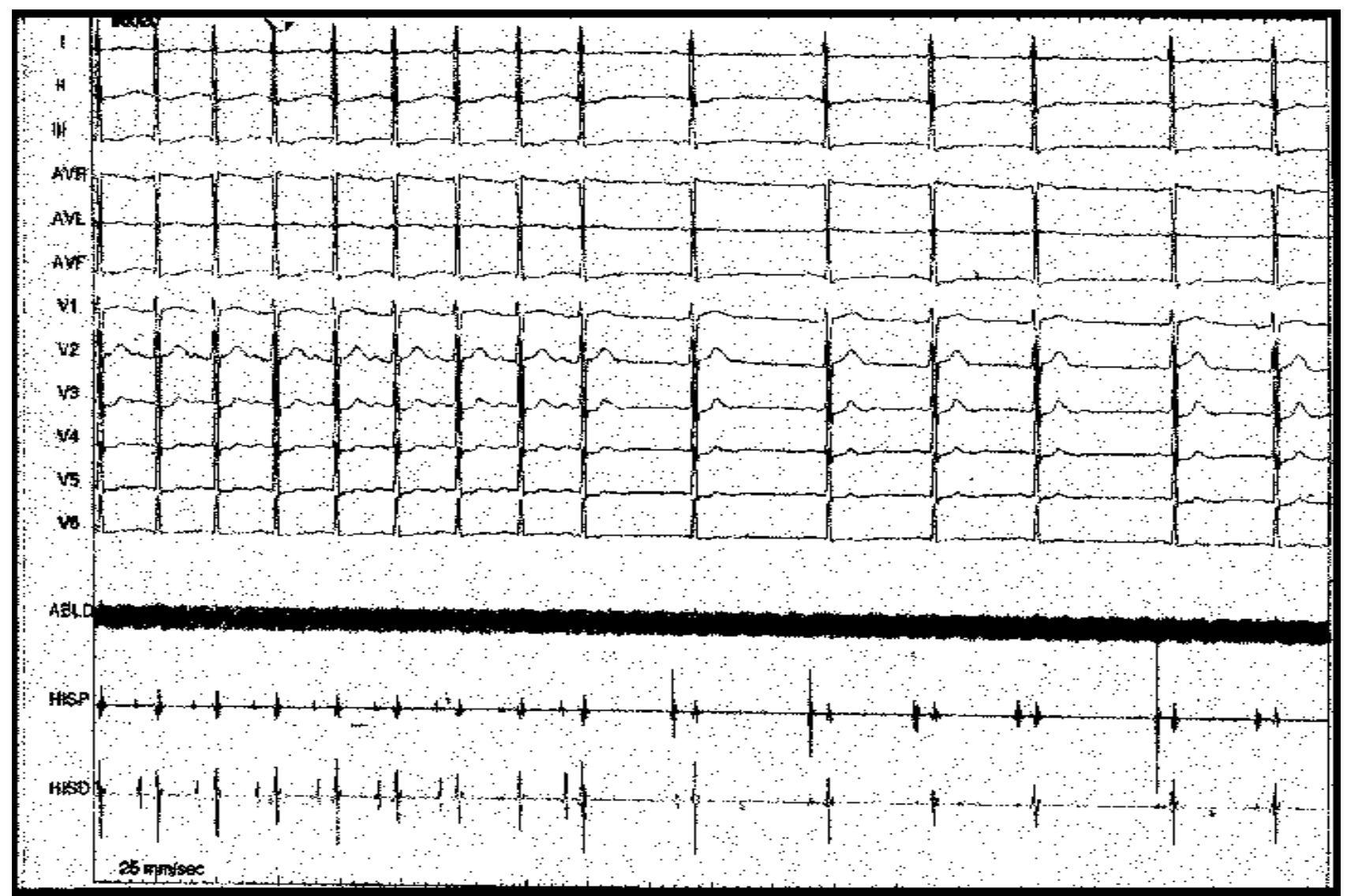

Fig. 1 - Eletrocardiograma com 12 derivações simultâneas, potencial do cateter de ablação (abld) e potencial proximal e distal da válvula tricúspide (hisp - hisd) no momento da redução dos ciclos cardíacos pela aplicação de radiofreqüência. 
$842,8 \pm 47,3 \mathrm{~ms}$, respectivamente. O confronto entre os resultados foi estatisticamente significante com um valor de $\mathrm{p}=0,000$. A tabela I apresenta a síntese dos resultados e a figura 3 em suas distribuições horárias.

\section{Discussão}

A taquicardia sinusal inapropriada é uma arritmia rara, de difícil controle clínico e de mecanismos de produção ainda relativamente obscuros. Em função dos sintomas produzidos, necessita de controle, sendo este, na maioria das vezes, inadequado em relação ao uso de drogas com ação bradicardizante sinusal. Frente a essas limitações e, com o advento da ablação por cateter com o uso de radiofreqüência, foi recentemente proposta uma nova forma de tratamento deste tipo de arritmia, baseada na produção de modificações da estrutura sinusal ou de suas regiões próximas, abolindo-se o foco arritmogênico e permitindo que novos grupos de células, com comportamento automático normal, assumam de forma definitiva a função de marcapasso principal $^{3,4}$.
Modificação do nódulo sinusal via cateter

\begin{tabular}{|c|c|c|c|}
\hline & $24 \mathrm{~h}$ & Vigília & Sono \\
\hline Pré & $520,9 \pm 89,1$ & $449 \pm 22,6$ & $612,1 \pm 81,7$ \\
\hline Imediato pós & $733,4 \pm 67,3$ & $723,2 \pm 49,7$ & $783,0 \pm 44,0$ \\
\hline 6 meses & $727,0 \pm 128,3$ & $599,4 \pm 98,0$ & $842,8 \pm 47,3$ \\
\hline $\mathrm{p}=$ & 0,000 & 0,000 & 0,000 \\
\hline
\end{tabular}

Em função da localização do foco arritmogênico e da resposta imediata ao procedimento, não podemos afastar a possibilidade do presente caso representar uma taquicardia atrial de caráter incessante, com foco próximo da estrutura sinusal. No entanto, a modificação na seqüência de ativação atrial, com aparecimento de um ritmo médio após a ablação, sugere que a energia liberada atingiu e modificou a estrutura sinusal (não permitindo a sua manifestação) ou que a mesma encontrava-se em profunda depressão automática e sem condições de assumir a condição de marcapasso principal, dúvidas, também, presentes quando analisamos os casos descritos em literatura ${ }^{3-5}$.

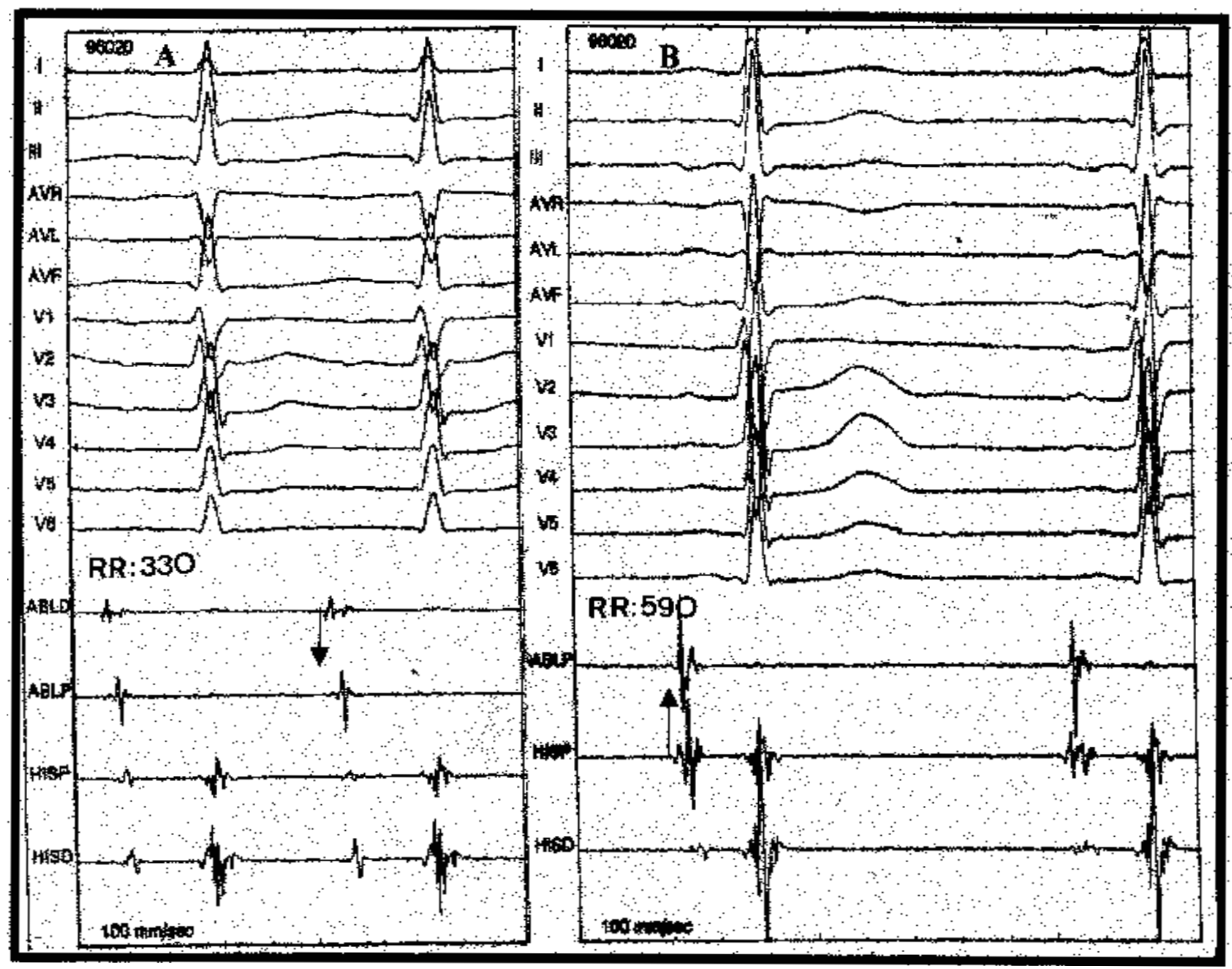

Fig. 2 - Seqüência de ativação atrial antes e após o procedimento (A e B, respectivamente). Nota-se que durante a taquicardia os átrios se ativam de forma semelhante à sinusal, ou seja, de átrio alto (ABLD - potencial distal do cateter de ablação - ABLP - potencial proximal do cateter de ablação) para átrio baixo (HISP - His proximal e HISD - His distal). Após a ablação há coincidência na ativação entre os potenciais de átrio alto (ABLP) e de válvula tricúspide, indicando o aparecimento de um ritmo atrial médio. 


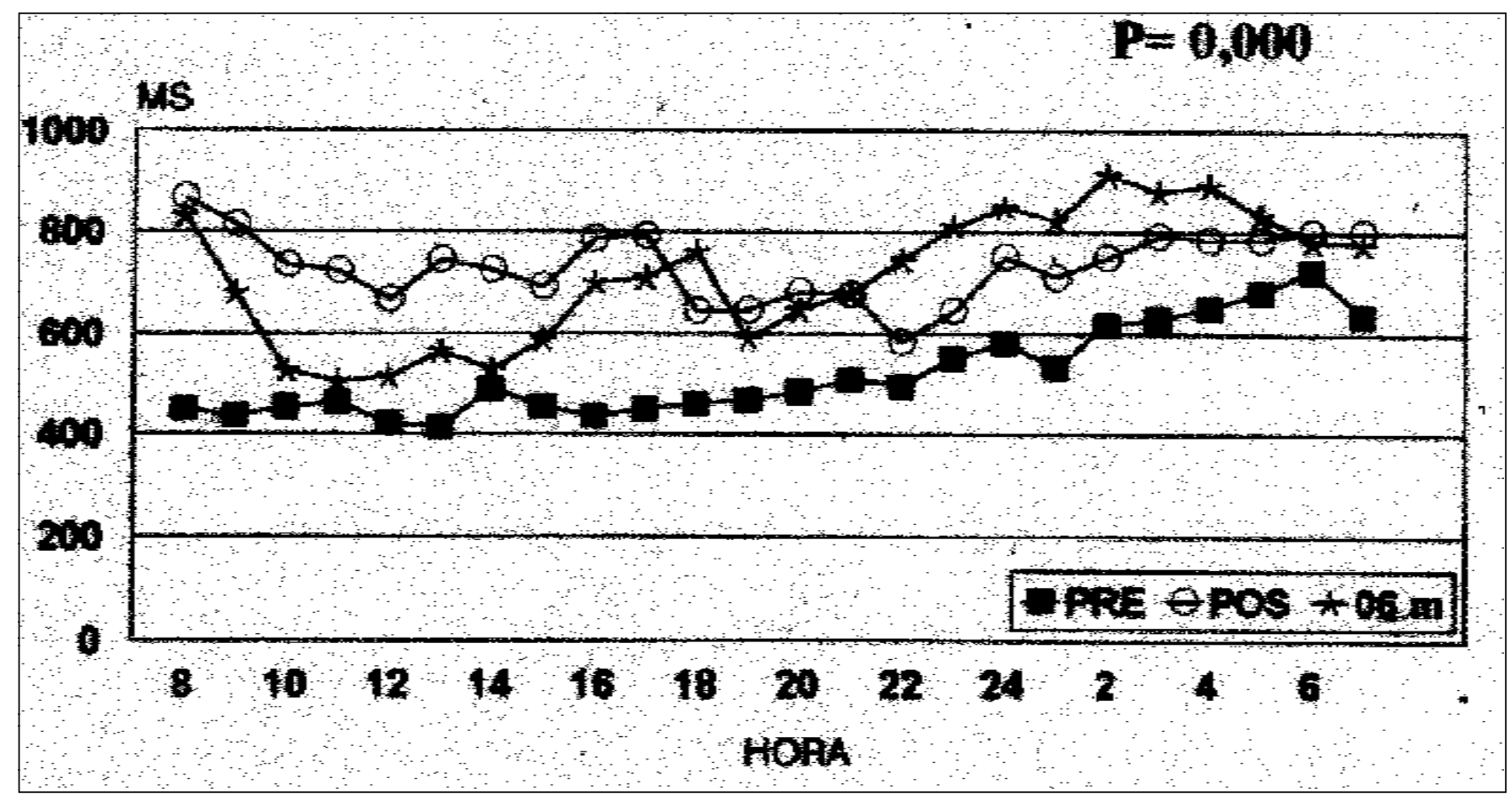

Fig. 3 - Gráfico com os ciclos cardíacos médios horários nas três situações avaliadas.

Embora com resultados iniciais e imediatos promissores, têm sido observadas recidivas da arritmia com um procedimento aparentemente de sucesso, necessitando-se de uma segunda intervenção. $\mathrm{O}$ retorno do evento arrítmico tem variado entre 20 a $25 \%$ em pequenas séries ${ }^{5,6}$, estando provavelmente relacionado com a existência de novos grupos de células, potencialmente anormais, que progressivamente adquirem as mesmas características eletrofisiológicas das previamente destruídas.

Apesar do controle imediato e tardio do distúrbio do ritmo, alguns pacientes continuam se mantendo sintomáticos, perdendo-se ao Holter a aparente correlação inicial entre os mesmos e os distúrbios eletrocardiográficos associados e sugerindo que, em algumas circunstâncias, o desenvolvimento de sintomas não decorra exclusivamente dos níveis de frequiência cardíaca elevada. A manutenção dos sintomas tem ocorrido em cerca de $30 \%$ dos pacientes que se submeteram a este tipo de procedimento com aparente sucesso.

Os resultados observados no presente caso foram considerados satisfatórios, atingindo-se os critérios adotados para aceitar, como de sucesso, o procedimento. Houve redução imediata significativa da freqüência cardíaca tanto no período global das $24 \mathrm{~h}$, como durante vigília e sono, havendo manutenção dos níveis normais após seis meses da intervenção. As curvas horárias dos ciclos cardíacos mostraram que os mesmos apresentaram um comportamento normal após a intervenção (fig. 3). Curiosamente, como pode ser observado na figura, os níveis máximos de freqüência cardíaca atingidos foram mais expressivos após seis meses da modificação, quando comparados aos observados imediatamente após a mesma. Além disso, notou-se também uma reatividade vagal noturna mais marcada, nos registros dos seis meses após o procedimento.

Apesar dos resultados favoráveis, a paciente vem se mantendo sintomática, com freqüentes episódios de dor precordial e cansaço. Novas gravações de Holter não puderam correlacionar tais sintomas com qualquer tipo de alteração eletrocardiográfica simultânea. $O$ procedimento não apresentou complicações tendo sido perfeitamente suportado pela paciente.

Em resumo, os resultados apresentados e os já observados em literatura demonstram que a modificação do nódulo sinusal por aplicação de energia de radiofreqüência via cateter, representa um novo procedimento seguro, sendo uma importante opção terapêutica para controle da freqüência cardíaca na taquicardia sinusal inapropriada. No entanto, o retorno a níveis apropriados de freqüência não produz, obrigatoriamente, recidiva dos sintomas.

\section{Referências}

1. Bauernfeind RA, Amat-Y-Leon F, Dhingra RC et al - Chronic nonparoxysmal sinus tachycardia in otherwise healthy persons. Ann Intern Med 1979; 91 : 702-10.

2. Scheinmann MM, Basu D, Hollenberg M - Electrophysiologic studies in patients with persistent atrial tachycardia. Circulation 1974; 50: 266-73.

3. Kay GN, Chong F, Epstein AE et al - Radiofrequency ablation for treatment of primary atrial tachycardias. J Am Coll cardiol 1993; 21: 901-9.

4. Waspe LE, Chien WW, Merillat JC, Stark SI - Sinus node modification using radiofrequency current in a patien with persistent inappropriate sinus tachycardia. PACE 1994; 17: 1569-76.

5. Taylor GW, Cooper RAS, Epstein AE et al - Long term follow-up of sinus node modification for treatment of innapropriate sinus tachycardia. PACE 1997; 20(Part II): 1203.

6. Shinbane JS, Lesh MD, Scheinmann MM et al - Long-term follow-up after radiofrequency sinus node modification for innapropriate sinus tachycardia. $\mathrm{J}$ Am Coll Cardiol 1997: 29(suppl A): 199A. 